\title{
EEG abnormalities with and without relation to severe hypoglycaemia in adolescents with type 1 diabetes
}

Received: 27 May 2004 / Accepted: 11 November 2004 / Published online: 1 March 2005

(C) Springer-Verlag 2005

\begin{abstract}
Aims/hypothesis: The aim of the present study was to identify whether adolescents with type 1 diabetes receiving modern multiple insulin injection therapy (MIT) have abnormal EEGs, and to elucidate possible correlations with a history of severe hypoglycaemia, poor metabolic control and nerve conduction defects. Methods: We investigated 35 patients (age 14-19 years) with disease duration 7.6 \pm 4.6 years, and 45 healthy control subjects. EEG spectral components were obtained from 15-min recordings in resting, awake subjects. Nerve conduction was measured bilaterally in motor and sensory fibres in the median, peroneal and sural nerves. Results: The EEGs of patients showed an increase in slow activity (delta and theta) and a reduction in alpha peak frequency, both of which were most pronounced in the frontal regions $(p<0.001)$. They also showed a decrease in fast activity, which was most pronounced bilaterally in the posterior temporal regions (alpha $p<0.001$, beta $p<0.01$, gamma $p<0.001$ ). A history of severe hypoglycaemia was correlated with a global increase in theta activity $(p<0.01-0.05)$. Poor metabolic control, measured as acute and long-term $\mathrm{HbA}_{1} \mathrm{c}$ levels, was correlated with an increase in delta activity and a decrease in alpha peak frequency. The decrease in fast activity in the temporal regions was a separate type of abnormality be-
\end{abstract}

\footnotetext{
L. Hyllienmark $\cdot$ J. Maltez $\cdot$ T. Brismar Department of Clinical Neurophysiology, Karolinska University Hospital, Stockholm, Sweden

L. Hyllienmark $(\bowtie)$

Department of Clinical Neurophysiology,

Karolinska University Hospital,

R2:01, 17176 Stockholm, Sweden

e-mail: lars.hyllienmark@karolinska.se

Tel.: +46-8-51772032

Fax: $+46-8-51774415$
}

\section{A. Dandenell · J. Ludvigsson}

Department of Paediatrics, Diabetes Research Centre, Faculty of Health Sciences, Linköping University Hospital, Linköping, Sweden cause it had a different distribution, and was not correlated with the increase in delta/theta power, poor metabolic control or with hypoglycaemia. Conclusions/interpretation: Recurrent severe hypoglycaemia and poor metabolic control are risk factors for EEG abnormalities in adolescents with type 1 diabetes receiving MIT treatment. In addition, we found pronounced abnormalities in the temporal regions that were not related to these risk factors.

Keywords Adolescents $\cdot$ EEG $\cdot$ Glycosylated haemoglobin · Hypoglycaemia · Metabolic control · Type 1 diabetes

Abbreviations AD: senile dementia of the Alzheimer's type - CMAP: compound muscle action potential - EMG: electromyogram - MCV: motor nerve conduction velocity MIT: modern multiple insulin injection therapy $\cdot \mathrm{SCV}$ : sensory nerve conduction velocity - SNAP: sensory nerve action potential

\section{Introduction}

Studies of brain function using different cognitive tests or EEG have shown an increased incidence of abnormalities in patients with type 1 diabetes. Poor metabolic control and repeated episodes of severe hypoglycaemia are recognised risk factors for cerebral dysfunction [1]. A decline in verbal and performance IQ has been found in children and adults with type 1 diabetes, and has been associated with either poor long-term metabolic control [2] or cumulative severe hypoglycaemia [3]. Early EEG studies revealed an increase in frontal slow activity and a decrease in alpha frequency in both children and adults with type 1 diabetes $[4,5]$. Quantitative EEG analysis has shown similar results, and patients with abnormal findings are characterised by either poor metabolic control [6] or recurrent severe hypoglycaemia $[7,8]$. In a recent EEG study of adults with wellcontrolled type 1 diabetes, we found a decrease in the power of faster frequencies, especially in the temporal regions, and an increase in the power of slower frequencies in 
the frontal regions in subjects without a history of frequent hypoglycaemic events [9].

It is well established that treatment with modern multiple insulin injection therapy (MIT) leading to improved metabolic control reduces nephropathy, retinopathy and peripheral neuropathy in patients with type 1 diabetes mellitus $[10,11]$. However, there is a risk that the tight control of blood sugar levels increases the incidence of severe hypoglycaemia [12]. Cognitive tests have indicated that recurrent hypoglycaemia during childhood may be even more harmful to the brain [13]. The purpose of the present study was to identify whether adolescents with type 1 diabetes using MIT have EEG abnormalities, and to elucidate possible correlations with various disease parameters, such as age of diabetes onset, degree of metabolic control, previous experience of severe hypoglycaemia and peripheral nerve dysfunction.

\section{Subjects and methods}

Subjects All 75 patients with type 1 diabetes aged $>14$ years attending the Paediatric Clinic at Linköping University Hospital (Linköping, Sweden) were considered for inclusion in the study. Of these, 36 patients had moved from the area or did not want to participate in the study. The EEGs of four patients were discarded due to significant artefacts during recording. This meant that the final study population consisted of 35 patients. Those included in the final study population were not significantly different to the patients who dropped out of the study in terms of age, duration of diabetes and level of metabolic control (data not shown). The study population included 16 females and 19 males with the following characteristics: age 17.1 \pm 1.7 years (range 14-19 years), disease duration $7.6 \pm 4.6$ years (range 2.0-17 years), age at onset 9.6 \pm 4.6 years (range 1.617 years), body length $170 \pm 8.5 \mathrm{~cm}$, body weight $70 \pm 10 \mathrm{~kg}$ and insulin dose $1.1 \pm 0.39 \mathrm{IE} \cdot \mathrm{kg}^{-1} \cdot \mathrm{day}^{-1}$. All patients were receiving intensive therapy, which involved the administration of insulin four to seven times daily, either by injection $(n=29)$ or an external subcutaneous infusion pump $(n=6)$. Each patient attended the clinic on one occasion for a clinical examination, EEG registration and peripheral nerve tests. The control group consisted of 45 healthy volunteers (21 females, 24 males) aged $16.8 \pm 1.6$ years (range 14-19 years) who were randomly recruited from the Swedish population register. The healthy control subjects were matched to the diabetic patients with respect to sex and age ( \pm 9 months), and did not have a history of neurological or chronic disease or medication. All subjects gave their informed consent prior to participation in the study. The protocol was approved by the Research Ethics Committee of the Faculty of Health Sciences, Linköping University Hospital.

Laboratory examinations Measurements of capillary blood glucose (Accutrend Mini; Boehringer Mannheim, Mannheim, Germany) and $\mathrm{HbA}_{1} \mathrm{c}$ (DCA 2000; Bayer, Leverkusen, Germany) levels, taken within 45 min of EEG registration, were $11 \pm 7.2 \mathrm{mmol} / \mathrm{l}$ (range $2.0-28 \mathrm{mmol} / \mathrm{l}$ ) and $7.2 \pm 1.2 \%$ (range $4.0-10 \%$ ) respectively. Data on $\mathrm{HbA}_{1} \mathrm{c}$ were collected retrospectively from the medical records of each patient and transformed in order to correspond to the values obtained by the method used in the present study. The normal range for $\mathrm{HbA}_{1} \mathrm{c}$ values was $3.2-6.0 \%$. Longterm metabolic control, defined as the mean of $\mathrm{HbA}_{1} \mathrm{c}$ for each year of the disease, was $6.7 \pm 0.8 \%$.

EEG recordings and spectral analysis EEGs were recorded and analysed using methods similar to those described earlier [9]. A digital EEG system (Nervus version 2.3, Taugagreining, Reykjavik, Iceland) was used with 22 Ag electrodes located according to the international 10-20 system (Fp1, Fp2, F7, F3, Fz, F4, F8, A1, T3, C3, Cz, C4, T4, A2, T5, P3, Pz, P4, T6, O1 and O2, with recording reference $\mathrm{Oz}$ ). Electrode impedances were $<5 \mathrm{k} \Omega$. The EEG signal was sampled at $256 \mathrm{~Hz}$, and cutoff frequencies of $<0.5$ and $>70 \mathrm{~Hz}$ were used. Recordings were performed over 15 min on awake, resting subjects, following a strict protocol, with an eyes open (10 s)/eyes closed (50 s) cycle repeated 15 times. Apart from instructions to open and close the eyes, the subjects were left completely undisturbed in order to standardise the conditions. Despite the repeated eye openings, some subjects became drowsy, and vertex sharp waves were observed in two of the patients during 30-40 s and in three control subjects during 20-50 s. Sleep spindles were not observed. The EEG recordings were performed between 16.00 and -18.00 hours on 34 patients and 38 control subjects; the remaining subjects were examined between 12.00 and -16.00 hours. If electrode artefacts became apparent during the beginning of the recording, the electrodes were adjusted and the recording session was restarted. Power parameters were calculated with reference to linked mastoids $(\mathrm{A} 1+\mathrm{A} 2)$. A bipolar transverse montage was applied in order to reduce the contamination of vertical eye movement artefacts in frontal delta activity. The entire recording was used for the quantitative analysis in order to avoid bias in the selection of EEG segments [14]. Segments containing significant artefacts [movement, electromyogram (EMG) artefacts, eye movement or blinks] were discarded, resulting in an EEG duration of $519 \pm 132 \mathrm{~s}$ (range $257-763 \mathrm{~s}$ ) from patients and $515 \pm 119 \mathrm{~s}$ (range 231-737 s) from control subjects. When a pulse or EMG artefact appeared during the recording in a single electrode (which occurred in $16 \%$ of the recordings, most often in frontal electrodes), the power data for that particular electrode were replaced with the values for the contralateral electrode. Power spectra were calculated by fast Fourier transformation (FFT) with a 4-s rectangular time window, from which an average spectrum for each channel was obtained in each subject. Comparison of the entire frequency spectra $(1-45 \mathrm{~Hz})$ in patients with those in control subjects showed the largest differences in power at 6-7, 9-11 and 18-22 Hz. For this reason, narrower segments of the power spectrum were defined within the conventional frequency bands as follows: $1-3 \mathrm{~Hz}$ ('delta'), 6-7 Hz ('theta'), 9-11 Hz ('alpha'), 18-22 Hz ('beta') and $38-42 \mathrm{~Hz}$ ('gamma'). These segments were chosen for 
statistical comparison as described previously [9]. The dominant frequency ('alpha peak') was measured in the range $7-13 \mathrm{~Hz}$ in order to detect a dominant peak slightly below the alpha band $(8-13 \mathrm{~Hz})$.

Statistical analysis Analysis of the EEG parameters of the control group showed an effect of both age and sex (data not shown). In order to compensate for these effects in the patients, a $z$ score was calculated for each EEG parameter and electrode position for each individual as (measured value-expected value)/standard deviation. The expected value was obtained by linear regression analysis of the effect of age in the control group, separately for males and females, and the standard deviation was obtained from the residual variance. Statistical analyses of differences between patients and control subjects ( $z$ scores) were performed using the Student's $t$-test for parameters with a normal distribution and the Mann-Whitney $U$-test for parameters with a non-normal distribution. The $\log _{10}$ of power for alpha, beta, gamma and delta was normally distributed in almost all electrode positions in bipolar and referential montage for both patients and control subjects (Lilliefors test). A non-normal distribution was observed in $38 \%$ of electrode positions for the $\log _{10}$ of theta power, and in $67 \%$ of electrode positions for the alpha peak frequency. The number of patients with $z$ scores $>2$ SD (number positive) or $<-2$ SD (number negative) was calculated for all parameters and electrode locations. The abnormality rate was calculated as (number positive-number negative)/(total number of patients), in order to obtain the net effect. Clinical parameters were analysed using a nonparametric ranking test (Spearman's rank correlation test), and group comparisons were made using the Mann-Whitney $U$-test. Multiple regression analysis was performed to determine the effects of sex, acute $\mathrm{HbA}_{1} \mathrm{c}$, and number of severe hypoglycaemic events on delta power. A $p$ value less than or equal to 0.05 was considered to be significant.

Electroneurography Studies of nerve conduction were performed using a Medelec Synergy system (Cephalon, Aarhus, Denmark) and surface electrodes as described previously [15]. Motor nerve conduction velocity (MCV) and compound muscle action potential (CMAP) amplitude were determined bilaterally in the median and peroneal nerves. The sensory nerve conduction velocity (SCV) and sensory nerve action potential (SNAP) amplitude were determined bilaterally in the median and sural nerves. The legs were warmed with heat pads for $10 \mathrm{~min}$ prior to the nerve conduction measurements. Skin temperature was measured with an accuracy of $0.1^{\circ} \mathrm{C}$ by a surface thermometer placed near the recording electrodes immediately after the nerve conduction measurement in each nerve. Skin temperature was $34 \pm 1.2^{\circ} \mathrm{C}$ (range $31-37^{\circ} \mathrm{C}$ ) on the dorsum of the foot (peroneal), $33 \pm 1.2^{\circ} \mathrm{C}$ (range $31-36^{\circ} \mathrm{C}$ ) on the back of the distal leg (sural), and $33 \pm 1.0^{\circ} \mathrm{C}$ (range $31-$ $37^{\circ} \mathrm{C}$ ) in the palm of the hand (median). All patients were examined by the same experienced technician. Complete nerve conduction studies were performed in all patients and the results were compared with previously published data in healthy control subjects [15]. The data were pooled in order to reduce the number of neurographic parameters. Abnormalities of the right side were correlated with abnormalities of the left side $(r=0.45-0.90, p<0.01)$. Dysfunction in peroneal MCV was correlated with dysfunction in median MCV $(r=0.72, p<0.0001)$, and dysfunction in sural SCV was correlated with dysfunction in median SCV $(r=0.43, p<0.001)$. After pooling the data, six parameters of nerve conduction were analysed: $\mathrm{MCV}, \mathrm{SCV}$, median CMAP, peroneal CMAP, median SNAP and sural SNAP. A $z$ score, corrected for height, was calculated for each conduction parameter as follows: (value in the patient-value in control subjects)/standard deviation. Patients had an average $z$ score of -1.4 for $\mathrm{MCV},-1.5$ for $\mathrm{SCV},-1.0$ for median CMAP ( $\log _{10}$ value), -1.2 for peroneal CMAP $\left(\log _{10}\right.$ value), -0.55 for median SNAP ( $\log _{10}$ value), and -0.70 for sural SNAP ( $\log _{10}$ value).

Clinical evaluation and examination A standard direct inquiry was made about hereditary neurological disease, ongoing medication, previous head injuries, presence of disease known to influence EEG, and peripheral nerve function or typical symptoms of peripheral neuropathy. None of the patients had a family history of peripheral neurological disease. Three patients were receiving medication for allergies, one for epilepsy and one for thyroid disease. Four patients had experienced commotio cordis, three had a history of febrile convulsions, and one patient contracted viral meningitis 10 years previously. Three patients smoked $>10$ cigarettes per day. Three patients had sensory symptoms (numbness in the lower legs). None of the patients had motor or autonomic symptoms, retinopathy or nephropathy. Tendon reflexes (bilaterally at the knees and ankles) and appreciation of vibration $(128 \mathrm{~Hz})$ bilaterally in the big toe were present in all patients. None of the patients had neuropathic ulcers. Blood pressure was measured using an inflatable cuff (width $12 \mathrm{~cm}$ ) with patients in the supine position after a 10-min rest. None of the patients were receiving anti-hypertensive medication, and none had a systolic blood pressure $>140 \mathrm{mmHg}$ or a diastolic blood pressure $>85 \mathrm{mmHg}$ in repeated examinations.

Previous history of hypoglycaemia and ketoacidosis According to medical records, 25 of the 35 patients had experienced severe hypoglycaemia (requiring help from another person) with or without unconsciousness. The median number of such episodes was nine (range of 1-49 episodes). On average, each girl had experienced 13 episodes of severe hypoglycaemia, whereas each boy had experienced six episodes $(p=0.07)$. Patients with severe hypoglycaemia had an earlier age of onset (8.1 years) and a longer duration of diabetes ( 9.2 years) than patients who had not experienced this complication (13 and 3.5 years respectively, $p<0.01)$. A history of severe hypoglycaemia was also correlated with higher acute and long-term $\mathrm{HbA}_{1} \mathrm{c}$ levels $(p<0.05)$, and reduced MCV and SCV $(p<0.01)$. The frequency of episodes of mild hypoglycaemia was 2-6 days per week in 12 patients, $1-4$ days per month in 22 patients and $<1$ day per month in one patient. According the present 
definition, hypoglycaemia in small children is always classified as severe. Ten patients had experienced hypoglycaemic events with unconsciousness; the median number of such events was $1-2$ (range $1-12$ events).

According to medical records, 11 patients had experienced one or more episodes of ketoacidosis. Ten of these patients had experienced one such episode, and one patient had experienced four episodes. Long-term $\mathrm{HbA}_{1} \mathrm{c}$ levels were positively correlated with disease duration $(p<0.01)$ and acute $\mathrm{HbA}_{1} \mathrm{c}$ levels $(p<0.001)$, and negatively correlated with age at onset $(p<0.01)$. Acute $\mathrm{HbA}_{1} \mathrm{c}$ levels were slightly higher in girls than in boys $(7.4 \mathrm{vs} 7.1 \%$, NS). $\mathrm{MCV}$ was negatively correlated with long-term $\mathrm{HbA}_{1} \mathrm{c}$ levels $(p<0.05)$ and with disease duration $(p<0.001)$. Acute blood sugar was positively correlated with $\mathrm{HbA}_{1} \mathrm{c}$ levels $(p<0.01)$, and SCV was positively correlated with MCV $(p<0.001)$.

\section{Results}

Different types of EEG abnormalities in adolescents with type 1 diabetes The spatial distribution of the EEG abnormalities induced by diabetes is illustrated in statistical brain maps that show the $p$ values of the $z$ scores of the patients (Fig. 1a, b). Slow activity (delta and theta) was increased bilaterally in the central and frontal regions, and showed a similar distribution in bipolar montage, with the largest increase seen in the medial frontal regions $(p<0.001$; Fig. 1b). There was a global reduction in fast activity; this was most pronounced bilaterally in the posterior temporal regions $(p<0.001, p<0.01$ and $p<0.001$ for alpha, beta and gamma power respectively). Abnormalities in these frequency bands were highly correlated (data not shown). The alpha peak frequency (defined as the dominant peak within the range $7-13 \mathrm{~Hz})$ was reduced $(p<0.01$ or $p<0.001)$ in all frontal regions. For example, the average alpha peak frequency in the right lateral frontal (F4-F8) region was $7.9 \mathrm{~Hz}$ in patients compared with $8.6 \mathrm{~Hz}$ in control subjects.

Abnormality rates and correlations between EEG abnormalities EEG abnormality rates were calculated in order to determine the incidence of abnormalities exceeding \pm 2 SD (see Subjects and methods). Except for those for theta power, the abnormality rates were highest in referential montage, where maximum values were $14-23 \%$ for delta power in the lateral frontal regions, $9-14 \%$ for theta power in the frontal and frontopolar regions, $11-17 \%$ for alpha power in the posterior temporal and occipital regions, and $26-34 \%$ for the alpha peak frequency in the frontopolar regions. Beta and gamma power had lower abnormality rates. A correlation analysis was performed in order to elucidate whether the increase in frontal slow activity was related to the decrease in temporal fast activity (Fig. 2); this was based on the fact that these regions showed the highest theta and alpha abnormality rates in bipolar montage. The analysis revealed that those patients with a pronounced decrease in alpha power in the posterior temporal region were different those with a large increase in theta power in
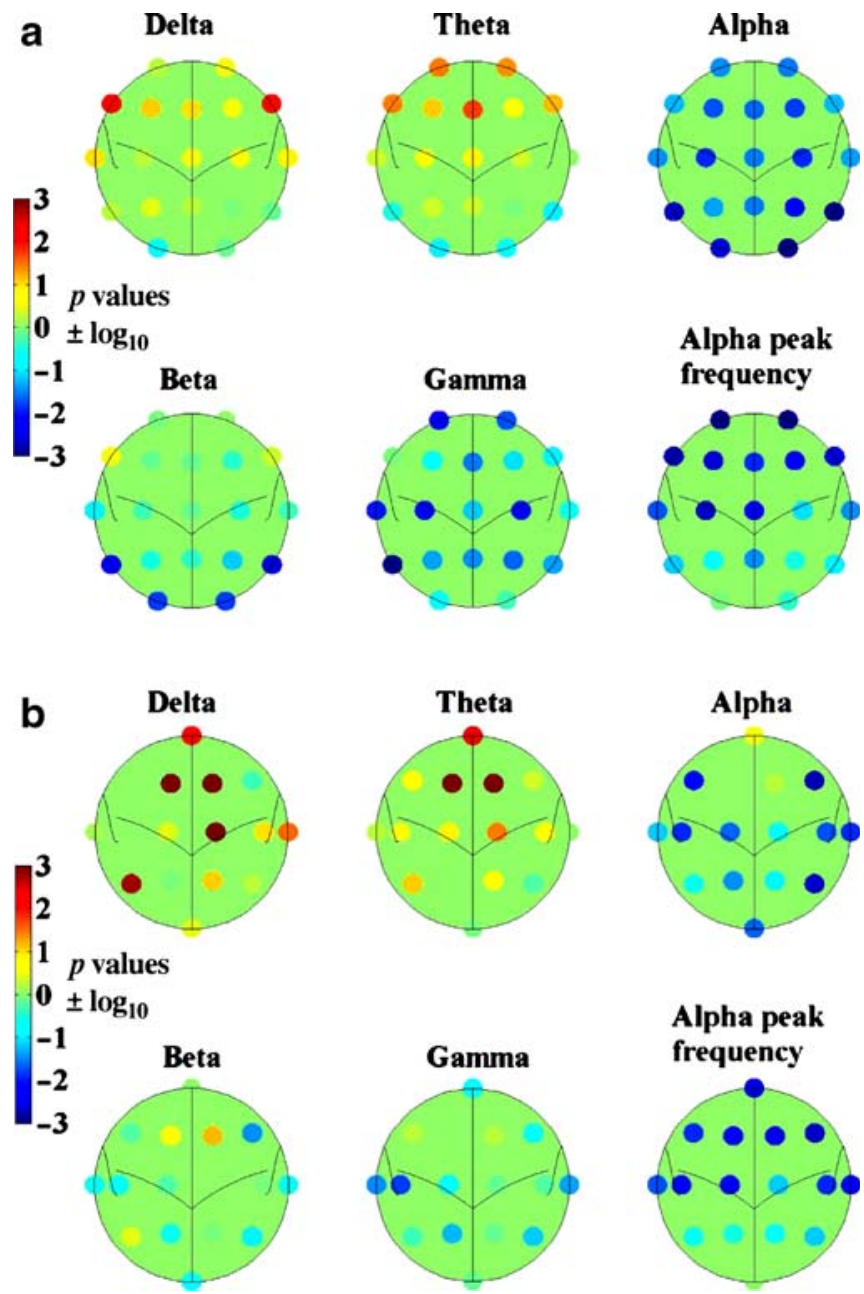

Fig. 1 Effect of diabetes on EEG spectral components in referential montage (a) and in bipolar montage (b). Brain maps showing the differences (see Subjects and methods) between patients with diabetes $(n=35)$ and healthy control subjects $(n=45)$. The different power bands are indicated. The colours represent the $\log _{10}$ of the $p$ values of the differences, i.e. 3 or -3 is equal to $p=0.001$. Red colours indicate an increase in power, whereas blue colours indicate a decrease in power. Electrodes were positioned according to the 10-20 system, and are fronto-polar in top, occipital in bottom, left temporal to the left, right temporal to the right, etc. Transverse bipolar EEG montage was applied in order to reduce the effect of eye movement artefacts in frontal leads

the frontal region, i.e. none of the patients had both alpha power abnormality in the posterior temporal region and theta power abnormality in the frontal region.

Effect of hypoglycaemic events on the EEG According to medical records, 25 patients had experienced one or more episodes of severe hypoglycaemia with or without unconsciousness. The effect of the number of severe hypoglycaemic episodes on the spectral parameters was studied with a ranking test. Theta power had a significant positive correlation in virtually all electrode positions $(r=0.36-0.51$, $p<0.01-0.05)$ except T5 ( $r=0.23$, NS) (Fig. 3). Delta power had a positive correlation in most of the frontal and central positions $(p<0.05$ or $p<0.01$ in five positions in referential montage). Alpha power was not correlated with the number 


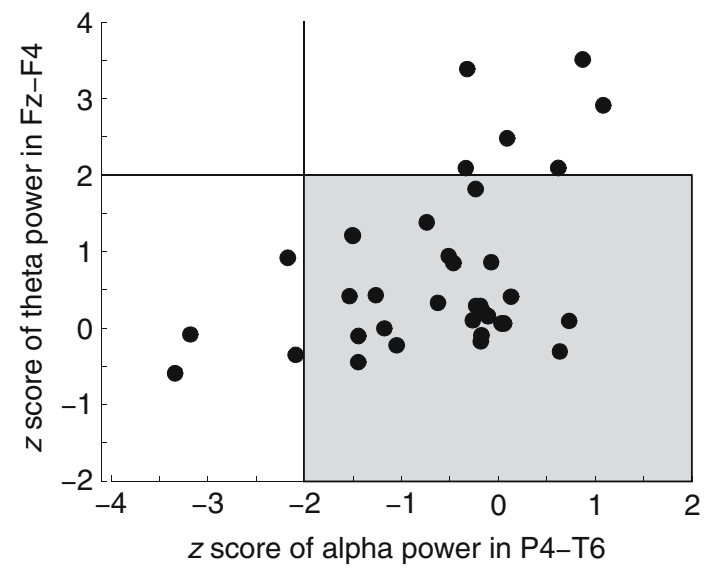

Fig. 2 Relationship between theta and alpha power abnormalities in patients with diabetes. Symbols show the $z$ scores for alpha power in the right parieto-temporal region (electrode P4-T6) and theta power in the right medial frontal region $(\mathrm{Fz}-\mathrm{F} 4)$ for each subject. The shaded area shows $z$ scores within \pm 2 SD of healthy control subjects. Note that no symbols are found in the upper left field, i.e. none of the patients had $z$ scores for alpha power $<-2$ SD and theta power $>2 \mathrm{SD}$

of hypoglycaemic events, whereas beta and gamma power had a positive correlation $(p<0.05)$ in six and five positions respectively. The alpha peak frequency was negatively correlated with the number of severe hypoglycaemic events in all electrode positions, and this effect was most pronounced in the right fronto-polar region $(\mathrm{Fp} 2, p<0.01)$. In this region, the alpha peak frequency was $8.1 \mathrm{~Hz}$ in those with severe hypoglycaemic events and $9.4 \mathrm{~Hz}$ in those without $(p<0.05)$. The alpha peak frequency in patients without a history of hypoglycaemia was not significantly different to that in healthy control subjects (e.g. $9.6 \mathrm{~Hz}$ in Fp2). Ten of the 25 patients who had experienced severe hypoglycaemia had also been unconscious on at least one occasion. In group comparison, these subjects were not different to those with severe hypoglycaemia without unconsciousness

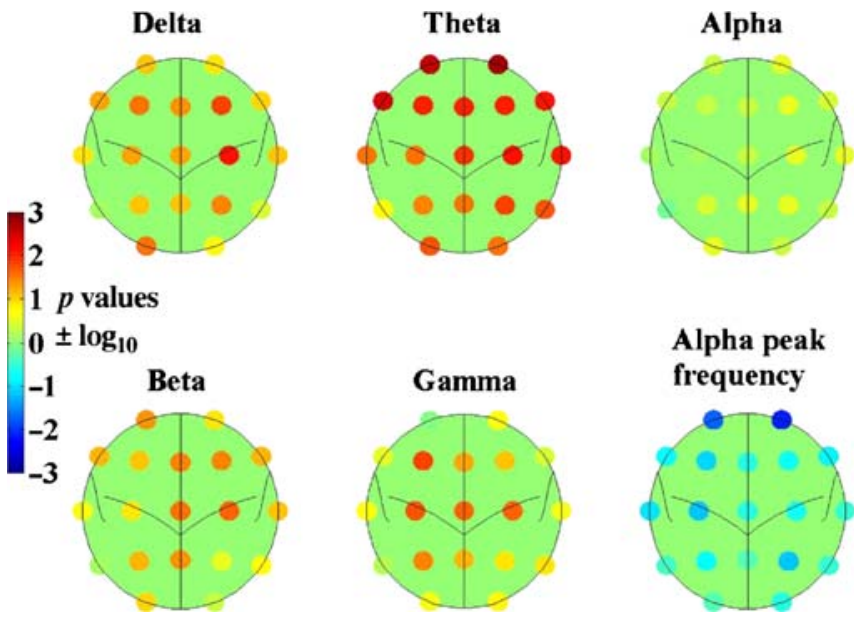

Fig. 3 Influence of the number of severe hypoglycaemic episodes on EEG power spectral parameters. The figure shows $p$ maps of the rank correlation test (Spearman rho). The electrode positions and colour coding are as in Fig. 1 in terms of any of the EEG parameters (data not shown). The frequency of mild hypoglycaemic events was not correlated with any of the abnormalities in the spectral parameters in the patients.

Effect of metabolic control Poor metabolic control, as indicated by acute and long-term $\mathrm{HbA}_{1} \mathrm{c}$ levels, had different effects on the EEG parameters. Acute $\mathrm{HbA}_{1} \mathrm{c}$ levels were correlated with a global increase in delta power, which was most pronounced in the frontal regions (maximum in F4, $p<0.001$ ). Long-term $\mathrm{HbA}_{1} \mathrm{c}$ had a significant negative correlation with the alpha peak frequency in eight out of 19 positions, which was also most pronounced in the frontal regions (maximum in $\mathrm{F} 3, p<0.01$ ). The other parameters were not affected, except for beta power, which showed a significant positive correlation with acute $\mathrm{HbA}_{1} \mathrm{c}$ levels in six out of 19 positions (data not shown). Acute blood glucose levels were not correlated with any of the parameters studied.

Effect of age, sex and other clinical parameters Age, age at onset of diabetes, duration of disease, BMI, insulin dose $\cdot \mathrm{kg}^{-1} \cdot \mathrm{day}^{-1}$, and number of episodes of ketoacidosis showed a significant effect on the EEG parameters in $<5 \%$ of the electrode positions. Nerve conduction properties (SCV and MCV) had a significant positive correlation with slow activity (delta and theta) in 10 and $7 \%$ of the electrode positions respectively. Response amplitudes (sural and median SNAP, and median and peroneal CMAP) showed a significant effect on the EEG spectral parameters in $<5 \%$ of electrode positions. In spite of the compensation for age and sex effects by the use of a $z$ score (see Subjects and methods), girls had higher values for delta power $(p<0.05$ 0.01 in 13 referential electrode positions). Multiple regression analysis was performed with delta power as a dependent variable, and sex, acute $\mathrm{HbA}_{1} \mathrm{c}$ levels and number of severe hypoglycaemic events as independent variables. When studied in the simple regression models, sex, acute $\mathrm{HbA}_{1} \mathrm{c}$ levels and number of hypoglycaemic events had a significant effect on delta power in 57,77 and $26 \%$ of the electrode positions respectively. However, in the multiple regression model, the effect was significant for the partial correlation in only 29,49 and $0 \%$ of the electrode positions respectively.

\section{Discussion}

EEG abnormalities were found in an unselected group of adolescents with type 1 diabetes who were receiving modern MIT treatment. The power of the faster frequencies (alpha, beta and gamma) was decreased, and this was most pronounced in the temporal regions. The slower frequencies (theta and delta) had an increased power, and the alpha peak frequency was reduced; both these abnormalities had maximum values in the frontal regions. Only the increase in slow activity and the decrease in alpha peak frequency could be correlated with disease history or laboratory data. The increase in theta activity was most strongly correlated 
with a history of severe hypoglycaemia, the increase in delta activity was most strongly correlated with high acute $\mathrm{HbA}_{1} \mathrm{c}$ levels and sex (stronger in girls), and the decrease in alpha peak frequency was most strongly correlated with high long-term $\mathrm{HbA}_{1} \mathrm{c}$ levels. The observed changes in the frequency spectrum and its regional distribution are in close agreement with those previously described in adult patients with type 1 diabetes [9]. Increased theta/delta power and decreased alpha power have been related to poor metabolic control and ketoacidosis in adolescents with type 1 diabetes [6]. Previous experience of severe hypoglycaemia has been related to increased theta/delta power and decreased alpha power in children [7], and to a global decrease in beta power in adult patients [8].

EEG slowing is usually observed in response to drowsiness. The recording procedure was standardised for all subjects and was designed to reduce spontaneous fluctuations in vigilance. Typical sleep patterns were infrequent and were observed to occur to the same extent in patients and control subjects. For these reasons it is unlikely that the observed increase in slow activity in the patients is related to a difference in vigilance between the two groups of subjects. Eye movements typically produce artefacts that are most pronounced in the frontal regions. This causes contamination of power in the delta range that is reduced with bipolar montage. Accordingly, by using this montage a more significant increase in frontal delta activity was observed in the patients, and this was more pronounced in girls. In order to adjust for the effect of age and sex, a $z$ score was utilised that was based on the control group. In spite of this, the multiple regression analysis showed that girls with diabetes did have an increase in delta power; however, the effect of acute $\mathrm{HbA}_{1} \mathrm{c}$ on delta power was more pronounced than the effect of sex.

The present analysis was performed in adolescents. Earlier studies have reported an increased risk of cognitive disturbances related to diabetes in this subpopulation of diabetes patients [13]. In this age group, the EEG shows maturational changes [16] that may obscure the detection of specific abnormalities. In young patients with diabetes EEG changes may be detected at blood glucose levels $<4 \mathrm{mmol} / \mathrm{l}$ (increase in slow activity), and an increase in frontocentral alpha activity has been described $<3 \mathrm{mmol} / 1$ [17]. Measurements made at the same time as the EEG recordings showed that blood glucose levels varied widely (2.0-28 mmol/l) and that the EEG parameters were not correlated with blood glucose levels during the recording. For these reasons it is not likely that the observed decrease in alpha activity in the present study is a reflection of low glucose levels during the EEG recording.

In small children all hypoglycaemic events were classified as severe (requiring assistance from other person). Using this definition only ten of the 35 patients had not experienced severe hypoglycaemia. Consequently, the number of episodes of severe hypoglycaemia may have been overestimated in patients with early age of onset. In spite of this ambiguity, hypoglycaemic events showed a strong and global correlation with theta power, whereas age of onset and diabetes duration did not. The ten patients who had experienced hypoglycaemic coma were not different to those without a history of coma with respect to the amount of theta activity or other parameters. This does not exclude the presence of short-lasting EEG effects of hypoglycaemic coma. However, investigation of this would require a prospective study design. As regards the influence of poor metabolic control on EEG, acute $\mathrm{HbA}_{1} \mathrm{c}$ levels were observed to have the strongest effect, showing a strong positive correlation with delta power. Long-term $\mathrm{HbA}_{1} \mathrm{c}$ levels most strongly affected the alpha peak frequency, and were negatively correlated with this EEG parameter. The increase in slow activity and the decrease in alpha peak frequency were also correlated with reductions in MCV and SCV. These observations are not surprising, since poor metabolic control is known to affect conduction properties [18]. The present data suggest that the effects of metabolic control are difficult to separate from those of severe hypoglycaemia since: (1) a history of severe hypoglycaemia is correlated with poor metabolic control; and (2) the EEG abnormalities correlated with severe hypoglycaemia and poor metabolic control had similar regional distributions with frontal maxima.

The findings of the present study indicate that the decrease in fast activity is a separate abnormality. In particular, alpha and beta power were decreased, with maximum reductions observed in the posterior temporal and occipital regions. The decrease in fast activity was not correlated with the increase in delta/theta power, neither was it correlated with poor metabolic control nor hypoglycaemia. The decrease in alpha power and faster frequencies in the posterior temporal region was the most significant abnormality in the present study. This finding is in agreement with a previous study on young adults with type 1 diabetes and a minor history of recurrent hypoglycaemia [9]. By calculating EEG abnormality rates we found that the incidence of decreased alpha peak frequency was highest in the frontal regions. This result was consistent with the distribution of the highest $p$ values in the Student's $t$-test. However, it should be noted that the abnormality rate is affected by small changes in the definition of the normal limits, and abnormalities that are present in group comparisons may be undetected. This may explain why the abnormality rates for delta power were highest in the lateral frontal regions, whereas the $p$ values were highest in the medial frontal regions.

The pathogenesis of the EEG abnormalities that were not related to hypoglycaemic events or to any of the other clinical variables may be linked to a more specific action of insulin and insulin receptors in the brain. Studies in the rat have shown that insulin receptors are widely distributed in the brain, with localisation densest in the olfactory bulbs and the limbic system, including the hippocampus and medial temporal cortex [19, for review see 20]. The insulin receptor guides the growth of retinal axons in Drosophila during the development of the visual system [21]. Insulindeficient rats have learning deficits and show alterations in the hippocampus at the cellular level in terms of decreased synaptic plasticity and increased neuronal apoptosis [22, 23]. In rats, an improvement in spatial memory following 
training was found to up-regulate the production of insulin receptor mRNA and increase the accumulation of the receptor protein in the hippocampus [24]. There is evidence that perturbation of insulin regulation contributes to the symptoms and pathogenesis of senile dementia of the Alzheimer's type (AD) [25]. Furthermore, subjects with type 2 diabetes are at increased risk of $\mathrm{AD}$, especially if they are treated with insulin [26].

Although cognitive function was not measured in the present study, there are reasons to believe that the EEG findings are relevant with regard to possible cortical dysfunction in diabetes. A shift in the EEG power spectrum, with a decrease in alpha/beta power and an increase in delta/theta power in the parietal and temporal regions, is typically observed in $\mathrm{AD}$, and there is a correlation between EEG abnormality and cognitive impairment [27]. Patients with type 1 diabetes may have various forms of cognitive dysfunction, including a decline in both verbal and performance IQ $[3,28]$. Whether repeated episodes of severe hypoglycaemia cause permanent cognitive impairment is unclear, as some studies have provided evidence for this $[3,29]$, whereas other studies have provided evidence to the contrary $[8,30,31]$. However, an association between 'psychomotor slowing' and poor metabolic control with peripheral neuropathy has been found in adult patients with type 1 diabetes $[1,32]$.

In conclusion, the present study demonstrates two kinds of EEG abnormalities in adolescents with type 1 diabetes receiving modern MIT treatment. A history of severe hypoglycaemia and poor metabolic control both contribute to the first kind of abnormalities, which have maximum values in the frontal regions. The second kind of abnormalities have maximally decreased values in the temporal regions and are not correlated with a history of severe hypoglycaemia, poor metabolic control, or the frontal abnormalities.

Acknowledgements This work was supported by grants from the Swedish Child Diabetes Foundation, the Torsten and Ragnar Söderberg's Foundations, and the Diabetes Strategy Project (Linköping University). The excellent technical assistance of G. Lundblad and L. Busta is gratefully acknowledged.

\section{References}

1. Ryan CM, Williams TM, Finegold DN, Orchard TJ (1993) Cognitive dysfunction in adults with type 1 (insulin-dependent) diabetes mellitus of long duration: effects of recurrent hypoglycaemia and other complications. Diabetologia 36:329-334

2. Schoenle EJ, Schoenle D, Molinari L, Largo RH (2002) Impaired intellectual development in children with type 1 diabetes: association with $\mathrm{HbA}_{1} \mathrm{c}$, age at diagnosis and sex. Diabetologia 45:108-114

3. Deary IJ, Crawford JR, Hepburn DA, Langan SJ, Blackmore LM, Frier BM (1993) Severe hypoglycemia and intelligence in adult patients with insulin-treated diabetes. Diabetes 42:341344

4. Izzo JL, Schuster DB, Engel GL (1953) The electroencephalogram of patients with diabetes mellitus. Diabetes 2:93-99

5. Eeg-Olofsson O (1977) Hypoglycemia and neurological disturbances in children with diabetes mellitus. Acta Paediatr Scand Suppl 270:91-96
6. Hauser E, Strohmayer C, Seidl R, Birnbacher R, Lischka A, Schober EJ (1995) Quantitative EEG in young diabetics. Child Neurol 10:330-334

7. Bjorgaas M, Sand T, Gimse R (1996) Quantitative EEG in type 1 diabetic children with and without episodes of severe hypoglycemia: a controlled, blind study. Acta Neurol Scand 93:398402

8. Howorka K, Pumprla J, Saletu B, Anderer P, Krieger M, Schabmann A (2000) Decrease of vigilance assessed by EEGmapping in type I diabetic patients with history of recurrent severe hypoglycaemia. Psychoneuroendocrinology 25:85-105

9. Brismar T, Hyllienmark L, Ekberg K, Johansson B-L (2002) Loss of temporal lobe beta power in young adults with type I diabetes mellitus. NeuroReport 13:1-5

10. The Diabetes Control and Complications Trial Research Group (1993) The effect of intensive treatment of diabetes on the development and progression of long-term complications in insulin-dependent diabetes mellitus. N Engl J Med 329:977986

11. Bojestig M, Arnqvist HJ, Hermansson G, Karlberg BE, Ludvigsson J (1994) Declining incidence of nephropathy in insulin-dependent diabetes mellitus. N Engl J Med 330:15-18

12. The Diabetes Control and Complications Trial Research Group (1991) Epidemiology of severe hypoglycemia in the Diabetes Control and Complications Trial. Am J Med 90:450-459

13. Ryan C, Vega A, Drash A (1985) Cognitive deficits in adolescents who developed diabetes early in life. Pediatrics 75:921927

14. Maltez J, Hyllienmark L, Nikulin V, Brismar T (2004) Time course and variability of power in different frequency bands of EEG during resting conditions. Clin Neurophysiol (in press)

15. Hyllienmark L, Ludvigsson J, Brismar T (1995) Normal values of nerve conduction in children and adolescents. Electroencephalogr Clin Neurophysiol 97:208-214

16. Eeg-Olofsson O (1971) The development of the electroencephalogram in normal adolescents from the age of 16 through 21 years. Neuropaediatrie 3:11-45

17. Bjorgaas M, Sand T, Vik T, Jorde R (1998) Quantitative EEG during controlled hypoglycemia in diabetic and non-diabetic children. Diabet Med 15:30-37

18. Hyllienmark L, Brismar T, Ludvigsson J (1995) Subclinical nerve dysfunction in children and adolescents with IDDM. Diabetologia 38:685-692

19. Kar S, Chabot JG, Quirion R (1993) Quantitative autoradiographic localization of $\left[{ }^{125} \mathrm{I}\right]$ insulin-like growth factor $\mathrm{I},\left[{ }^{125} \mathrm{I}\right]$ insulin-like growth factor II, and $\left.{ }^{125} \mathrm{I}\right]$ insulin receptor binding sites in developing and adult rat brain. J Comp Neurol 333: 375-397

20. Unger JW, Livingston JN, Moss AM (1991) Insulin receptors in the central nervous system: localization, signalling mechanisms and functional aspects. Prog Neurobiol 36:343-362

21. Song J, Wu L, Chen Z, Kohanski RA, Pick L (2003) Axons guided by insulin receptor in Drosophila visual system. Science 300:502-505

22. Kamal A, Biessels GJ, Duis SEJ, Gispen WH (2000) Learning and hippocampal synaptic plasticity in streptozotocin-diabetes rats: interaction of diabetes and ageing. Diabetologia 43:500 506

23. Li Z-G, Zhang W, Grunberger G, Sima AAF (2002) Hippocampal neuronal apoptosis in type 1 diabetes. Brain Res 946:221-231

24. Zhao W, Chen H, Xu H et al (1999) Brain insulin receptors and spatial memory. J Biol Chem 274:34893-34902

25. Craft S, Stennis Watson G (2004) Insulin and neurodegenerative disease: shared and specific mechanisms. Lancet Neurol $3: 169-178$

26. Ott A, Stolk RP, van Harskamp F, Pols HAP, Hofman A, Breteler MMB (1999) Diabetes mellitus and the risk of dementia: the Rotterdam Study. Neurology 53:1937-1942 
27. Brenner RP (1999) EEG and dementia. In: Niedermayer E, Lopez Da Silva F (eds) Electroencephalography basic principles, clinical applications, and related fields. Williams \& Wilkins, Baltimore, pp 349-359

28. Langan SJ, Deary IJ, Hepburn DA, Frier BM (1991) Cumulative cognitive impairment following recurrent severe hypoglycaemia in adult patients with insulin-treated diabetes mellitus. Diabetologia 34:337-344

29. Wredling R, Levander S, Adamson U, Lins PE (1990) Permanent neuropsychological impairment after recurrent episodes of severe hypoglycaemia in man. Diabetologia 33:152-157
30. The Diabetes Control and Complications Trial Research Group (1996) Effects of intensive diabetes therapy on neuropsychological function in adults in the Diabetes Control and Complications Trial. Ann Intern Med 124:379-388

31. Kramer L, Fasching P, Madl C et al (1998) Previous episodes of hypoglycemic coma are not associated with permanent cognitive brain dysfunction in IDDM patients on intensive insulin treatment. Diabetes 47:1909-1914

32. Ryan CM, Williams TM, Orchard TJ, Finegold DN (1992) Psychomotor slowing is associated with distal symmetrical polyneuropathy in adults with diabetes mellitus. Diabetes 41: $107-113$ 